\title{
Study protocol for a systematic review of evidence for lifestyle interventions targeting smoking, sleep, alcohol/other drug use, physical activity, and healthy diet in people with bipolar disorder
}

Frances J. Kay-Lambkin ${ }^{1,2^{*}}$, Louise Thornton ${ }^{1}$, Julia M. Lappin ${ }^{3}$, Tanya Hanstock ${ }^{2,10}$, Louisa Sylvia ${ }^{4}$, Felice Jacka ${ }^{5}$, Amanda L. Baker ${ }^{2}$, Michal Berk ${ }^{5}$, Phillip B. Mitchell ${ }^{3}$, Robin Callister ${ }^{6}$, Naomi Rogers ${ }^{7}$, Stephanie Webster ${ }^{1}$, Simon Dennis ${ }^{10}$, Christopher Oldmeadow ${ }^{8}$, Andrew MacKinnon ${ }^{9}$, Christopher Doran ${ }^{8}$, Alyna Turner ${ }^{2,5}$ and Sally Hunt ${ }^{1}$

\begin{abstract}
Background: People with bipolar disorder (BD) have a mortality gap of up to 20 years compared to the general population. Physical conditions, such as cardiovascular disease (CVD) and cancer, cause the majority of excess deaths in psychiatric populations and are the leading causes of mortality in people with BD. However, comparatively little attention has been paid to reducing the risk of physical conditions in psychiatric populations. Unhealthy lifestyle behaviors are among the potentially modifiable risk factors for a range of commonly comorbid chronic medical conditions, including CVD, diabetes, and obesity. This systematic review will identify and evaluate the available evidence for effective interventions to reduce risk and promote healthy lifestyle behaviors in BD.

Methods/design: We will search MEDLINE, Embase, PsychINFO, Cochrane Database of Systematic Reviews, and CINAHL for published research studies (with at least an abstract published in English) that evaluate behavioral or psychosocial interventions to address the following lifestyle factors in people with BD: tobacco use, physical inactivity, unhealthy diet, overweight or obesity, sleep-wake disturbance, and alcohol/other drug use. Primary outcomes for the review will be changes in tobacco use, level of physical activity, diet quality, sleep quality, alcohol use, and illicit drug use. Data on each primary outcome will be synthesized across available studies in that lifestyle area (e.g., tobacco abstinence, cigarettes smoked per day), and panel of research and clinical experts in each of the target lifestyle behaviors and those experienced with clinical and research with individuals with BD will determine how best to represent data related to that primary outcome. Seven members of the systematic review team will extract data, synthesize the evidence, and rate it for quality. Evidence will be synthesized via a narrative description of the behavioral interventions and their effectiveness in improving the healthy lifestyle behaviors in people with BD.

(Continued on next page)
\end{abstract}

\footnotetext{
* Correspondence: f.kaylambkin@unsw.edu.au

${ }^{1}$ National Health and Medical Research Council Centre for Research

Excellence in Mental Health and Substance Use, National Drug and Alcohol

Research Centre, University of New South Wales, Sydney, Australia

${ }^{2}$ Priority Research Centre for Translational Neuroscience and Mental Health,

The University of Newcastle, Newcastle, Australia

Full list of author information is available at the end of the article
} 
(Continued from previous page)

Discussion: The planned review will synthesize and evaluate the available evidence regarding the behavioral or psychosocial treatment of lifestyle-related behaviors in people with BD. From this review, we will identify gaps in our existing knowledge and research evidence about the management of unhealthy lifestyle behaviors in people with BD. We will also identify potential opportunities to address lifestyle behaviors in $\mathrm{BD}$, with a view to reducing the burden of physical ill-health in this population.

Systematic review registration: PROSPERO CRD42015019993

Keywords: Bipolar disorder, Risk, Prevention, Mania, Depression, Tobacco, Smoking, Physical activity, Sleep, Diet, Weight loss, Alcohol/other drug use, Interventions, Treatment, Morbidity, Mortality

\section{Background}

People with mental illness have a reduced life expectancy of between 12 and 20 years compared to the general population $[1,2]$. To date, much of the attention on this issue has focused on the increased risks of suicide in people with mental illness, which has been shown to account for about $20 \%$ of the premature deaths in populations with a mental illness. Comparatively little attention has been paid to reducing the risk of physical conditions, which account for $80 \%$ of excess deaths in psychiatric populations [2].

A particular focus on bipolar disorder (BD) is important. In $\mathrm{BD}$, reduced life expectancy in comparison with the general population ranges from 11 to 20 years depending on the age of onset of the disorder [3]. The reasons for this disparity are complex and multi-faceted, involving genetic, biological, environmental, psychological, and social factors. Cardiovascular disease (CVD) diagnoses occur 6 years earlier for those with BD than for depression and 15 years ahead of the general population [4]. Many factors are attributed to this, including the significant and adverse effects of medication for BD, such as weight gain, metabolic syndrome, high cholesterol, hypertriglyceridemia, and other cardiometabolic problems [5]. Medications commonly prescribed in BD-anti-manic, antipsychotic, and antidepressant treatments-can negatively contribute through adverse side effects including weight gain, sedation, and disordered glucose tolerance [6]. In addition, the phases of $\mathrm{BD}$ mean that people can oscillate between intense periods of severe depression (with high risk of suicide) followed by intense periods of activity and irritability, masking a cycle of agitation, sleep, and physical exhaustion that can have a significant impact on confidence, selfefficacy, and health-related decisions [7]. Up to $65 \%$ of people with BD report current tobacco use [8] and smoke at 2.6 times the rate of people with major depressive disorder (MDD) and 6.3 times more than the general population [9]. Smoking is independently associated with suicide risk, poorer response to treatment, and structural brain changes [10]. Physical inactivity has been identified as an independent predictor of premature mortality among people with $\mathrm{BD}$ and significantly increases their risk of CVD [11]. In a study of 1046 Australian women, poor diet quality was associated with twice the odds for BD [12]. In another study comparing 2032 participants with $\mathrm{BD}$ in the general population, having BD was associated with significantly poorer eating behaviors, including fewer daily meals and difficulty obtaining or cooking food, as well as increased appetite and caloric intake [13]. Complicating this and supporting an integrated approach, people at risk for one adverse health behavior are at greater risk for others [14]. There is often poor integration between mental health and physical health services internationally and well-documented concerns by people with mental illness using existing services, who report inequity of access to physical-health-related interventions and support [9]. Together, the periods of being unwell with a psychiatric illness, combined with side effects of many medications, may contribute to the inequality in physical wellness for clients with mental health issues and the general population.

In order to reduce the mortality gap between people with $\mathrm{BD}$ and the general population, greater understanding is required of how best to assist them and their clinicians to address the potentially modifiable lifestyle behaviors associated with this gap $[15,16]$. Recently, the International Society for Bipolar Disorder (ISBD) developed consensus guidelines for monitoring of adverse side effects associated with commonly prescribed medications for the disorder [17]. The aim for these guidelines was to increase the acknowledgement and focus of health care providers on the physical health comorbidities germane to the management of BD [17]. Whilst this is critically important to the field, reducing the medication load for people with BD is just one strategy in addressing the mortality gap associated with the condition [18].

Of additional importance is to identify acceptable and effective interventions to improve the health outcomes of people with $\mathrm{BD}$. There is increasing recognition of the importance of supporting all people with mental health problems to focus on lifestyle factors, such as smoking 
cessation, in addition to symptom management [19]. However, the nature of $\mathrm{BD}$ may mean that additional strategies are indicated to ensure safety and well as efficacy of these approaches. In contrast to other mental disorders, for example, regulation and structure are critical in BD for both symptom and lifestyle management. For example, when depressed, strategies need to encourage stimulation and activation; however, when manic, the opposite is true [20]. Key withdrawal symptoms of nicotine are insomnia and increased appetite, with standard behavioral programs recommending increased physical activity to cope with cravings, stress, and weight gain [21]. Sleep disturbance, increased appetite, and excessive exercise are all critical warning signs for a relapse to BD [22], and thus careful strategies for self-regulation may be a unique feature of any program that recommends changing these behaviors in people with BD. To date, no systematic review has examined these issues or the available evidence to support lifestyle management in people with BD specifically.

The objective of this review is to systematically identify and evaluate the literature regarding lifestyle behavioral interventions among people with $\mathrm{BD}$. We aim to synthesize the available evidence regarding the management of unhealthy lifestyle behaviors and the promotion of health behaviors in people with BD, with regard to the impact of such interventions on health, symptoms, and related outcomes. Specifically, we aim to answer the following questions:

a) Which, if any, interventions have demonstrated effectiveness in the treatment of tobacco use, sleep-wake disturbances, alcohol/other drug use, or in encouraging healthy eating or healthy levels of physical activity for people with BD?

b) Do any integrated evidence-based interventions exist that target a range of lifestyle behaviors within one package for people with $\mathrm{BD}$ ? If so, do these integrated treatments produce improvements across all of the treatment targets?

c) Have any lifestyle-based interventions been tested in people with BD that incorporate eHealth (and related technologies)? If so, how have outcomes compared with face-to-face delivered interventions?

\section{Methods}

This systematic review is registered with the International Prospective Register of Systematic Reviews (PROSPERO, http://www.crd.york.ac.uk/PROSPERO, registration number: CRD42015019993). The protocol has been written according to the Preferred Reporting Items for Systematic Reviews and Meta-Analyses-Protocol (PRISMA-P) recommendations for systematic review protocols [23], and the findings will be reported using PRISMA guidelines [24].

\section{Criteria for study inclusion}

To be eligible for inclusion in the planned review, articles will be required to describe an intervention conducted in people with BD aged 18 years and over. Any type of behavioral or psychosocial intervention will be considered; however, articles must report at least one behavioral health or lifestyle outcome (e.g., smoking, alcohol or other substance use, diet, physical activity, sleep). Additionally, eligible articles will be required to report their results for participants with bipolar disorder separately to any other participants in the study, be published in a peer-reviewed journal, and published in English (at least an abstract). Randomized trials as well as uncontrolled trials including cohort, historical, and case-control studies will be considered. Our primary outcomes of interest will be the following lifestyle behavior outcomes: tobacco use, physical activity, diet quality, sleep quality, alcohol use, cannabis use, and illicit drug use from baseline to the last available follow-up. Secondary outcomes of interest will include participants' psychiatric symptoms and physical health outcomes such as changes in quality of life scores, depression, manic symptoms, body mass index, and other CVD risk factors from baseline to the last available follow-up as available.

\section{Search strategy for identification of studies}

We will search the following electronic databases to identify potentially eligible studies for inclusion in the review: MEDLINE, Embase, PsychINFO, Cochrane Database of Systematic Reviews, and CINAHL. The search will be limited to human research and those with at least an abstract written in English. No date restrictions will be placed on the searches, and search terms will be intentionally kept broad and general to increase the likelihood of capturing potentially eligible studies. An example of the proposed search strategy for MEDLINE is outlined in Table 1.

\section{Data extraction}

Following de-duplication of the search results, two team members (LT and FK-L) will independently review the titles and abstracts of all returned articles to identify studies that potentially meet the inclusion criteria. Potentially eligible articles will then be reviewed in full by two team members against the eligibility criteria (LT and FK-L). Any disagreement between the team members regarding eligibility will be resolved through discussion with a third reviewer (LS). Reasons why studies were excluded from this phase of the search will be recorded.

Trial reports (e.g., from clinical trial registries) for included studies will be reviewed to determine whether outcomes of interest were assessed but not reported in the identified publication. For older studies where trial registration was not mandatory, authors will be contacted to 
Table 1 Search strategy using MEDLINE 1946 to present with daily update

\begin{tabular}{|c|c|c|}
\hline Number & Searches & Results \\
\hline 1 & Bipolar Disorder/ & 33,501 \\
\hline 2 & bipolar*.tw. & 43,114 \\
\hline 3 & affective disorder*.mp. or Mood Disorders/ & 24,504 \\
\hline 4 & manic*.tw. & 9456 \\
\hline 5 & mania.tw. & 7439 \\
\hline 6 & hypomani*.tw. & 2075 \\
\hline 7 & $\begin{array}{l}\text { (mixed adj (state* or episode*)).mp. [mp = title, } \\
\text { abstract, original title, name of substance word, } \\
\text { subject heading word, keyword heading word, } \\
\text { protocol supplementary concept word, rare disease } \\
\text { supplementary concept word, unique identifier] }\end{array}$ & 1096 \\
\hline 8 & Cyclothymic Disorder/ & 565 \\
\hline 9 & cyclothymi*.tw. & 751 \\
\hline 10 & rapid next cycl*.tw. & 0 \\
\hline 11 & schizoaffective.tw. & 4195 \\
\hline 12 & $\begin{array}{l}\text { Affective Disorders, Psychotic/or Psychotic } \\
\text { Disorders/ }\end{array}$ & 40,311 \\
\hline 13 & unipolar*.tw. & 8387 \\
\hline 14 & $\begin{array}{l}1 \text { or } 2 \text { or } 3 \text { or } 4 \text { or } 5 \text { or } 6 \text { or } 7 \text { or } 8 \text { or } 9 \text { or } 10 \text { or } 11 \\
\text { or } 12 \text { or } 13\end{array}$ & 117,677 \\
\hline 15 & $\begin{array}{l}\text { Smoking/or Marijuana Smoking/or Smoking } \\
\text { Cessation/ }\end{array}$ & 137,906 \\
\hline 16 & "Tobacco Use"/ & 394 \\
\hline 17 & (smok* or tobacco or cigarette* or nicotine).tw. & 244,392 \\
\hline 18 & exp Alcohol Drinking/ & 55,319 \\
\hline 19 & alcoholic intoxication/or alcoholism/ & 78,084 \\
\hline 20 & (alcohol* or drinking).tw. & 281,327 \\
\hline 21 & marijuana abuse/or exp opioid-related disorders/ & 24,563 \\
\hline 22 & Cannabis/ & 7067 \\
\hline 23 & designer drugs/or exp street drugs/ & 10,732 \\
\hline 24 & exp Sleep/ & 64,880 \\
\hline 25 & sleep.tw. & 104,983 \\
\hline 26 & Exercise/or Exercise Therapy/ & 101,251 \\
\hline 27 & Physical Exertion/ & 53,748 \\
\hline 28 & Motor Activity/ & 83,896 \\
\hline 29 & Sports/ & 25,382 \\
\hline 30 & $\begin{array}{l}\text { (Physical Education and Training).mp. [mp = title, } \\
\text { abstract, original title, name of substance word, } \\
\text { subject heading word, keyword heading word, } \\
\text { protocol supplementary concept word, rare disease } \\
\text { supplementary concept word, unique identifier] }\end{array}$ & 12,568 \\
\hline 31 & exp Diet Therapy/ & 44,656 \\
\hline 32 & (diet* adj5 (health* or weight*)).tw. & 26,065 \\
\hline 33 & (calorie adj5 (control or reduc* or restrict*)).tw. & 3102 \\
\hline 34 & food choice*.tw. & 2516 \\
\hline 35 & (exercise* or sport*).tw. & 232,095 \\
\hline 36 & (physical activit* or physical inactivit*).tw. & 62,477 \\
\hline
\end{tabular}

Table 1 Search strategy using MEDLINE 1946 to present with daily update (Continued)

\begin{tabular}{|c|c|c|}
\hline 37 & nutrition*.tw. & 179,639 \\
\hline 38 & Sedentary Lifestyle/ & 4056 \\
\hline 39 & (sedentary adj5 (lifestyle or behavio? $\left.\left.{ }^{*}\right)\right)$.tw. & 4236 \\
\hline 40 & (weight adj (loss or reduc* or gain* or change*)).tw. & 103,986 \\
\hline 41 & (drug adj (use* or abus*)).tw. & 56,661 \\
\hline 42 & (diet* adj5 quality).tw. & 4443 \\
\hline 43 & $\begin{array}{l}15 \text { or } 16 \text { or } 17 \text { or } 18 \text { or } 19 \text { or } 20 \text { or } 21 \text { or } 22 \text { or } 23 \\
\text { or } 24 \text { or } 25 \text { or } 26 \text { or } 27 \text { or } 28 \text { or } 29 \text { or } 30 \text { or } 31 \text { or } \\
32 \text { or } 33 \text { or } 34 \text { or } 35 \text { or } 36 \text { or } 37 \text { or } 38 \text { or } 39 \text { or } 40 \\
\text { or } 41 \text { or } 42\end{array}$ & $1,374,954$ \\
\hline 44 & random*.tw. & 711,373 \\
\hline 45 & $\begin{array}{l}\text { cross-over studies/or double-blind method/or } \\
\text { random allocation/or single-blind method/ }\end{array}$ & 248,804 \\
\hline 46 & Randomized Controlled Trial/ & 404,345 \\
\hline 47 & Controlled Clinical Trial/ & 89,970 \\
\hline 48 & trial.tw. & 368,603 \\
\hline 49 & groups.tw. & $1,376,585$ \\
\hline 50 & (evaluation* or treatment* or intervention*).tw. & $4,060,728$ \\
\hline 51 & 44 or 45 or 46 or 47 or 48 or 49 or 50 & $5,446,183$ \\
\hline 52 & 14 and 43 and 51 & 6410 \\
\hline 53 & limit 52 to english language & 5828 \\
\hline
\end{tabular}

determine whether data on the outcomes of interest to the review are available for provision to the systematic review team. The abstracts of included studies not published in full using English will be reviewed for potential for data extraction.

Based on the results of the eligibility step, included studies will be divided into categories, according to the primary health behavior or lifestyle target of the intervention (tobacco, physical activity, diet quality, sleep-wake disturbance, and alcohol/other drugs). Included studies on weight loss interventions will be reviewed for information about the primary target of behavior within the weight loss intervention (e.g., physical activity, diet). We expect that, within each lifestyle intervention/behavior, a range of outcomes will be reported (e.g., minutes or hours of moderate or vigorous activity per day or week). We will extract data related to all reported outcomes for each included study for this review. Studies reporting more than one behavioral outcome will contribute data to all relevant intervention analyses.

Data will be extracted from all eligible studies using a data extraction form specifically developed for this study (see Additional file 1). Extracted data will include study design, study setting, sample size, study population and participant demographics (including diagnoses), baseline characteristics (including phase of bipolar disorder), recruitment and retention rates (including completion), 
details of the intervention, details of health behavior outcomes (all reported timepoints), details of other reported study outcomes (e.g., quality of life, BD symptoms), indicators of acceptability to participants, and information for the assessment of the risk of bias. All reported trial outcomes related to the lifestyle behavior targeted by the intervention will be extracted in the event that a study does not report on the primary outcome for the review but reports on a structurally/conceptually similar outcome related to the primary outcome (possible reporting bias).

Seven review authors (LT, JL, FJ, TH, AT, SH, and FK-L) will independently extract data from eligible studies using the standardized form, with discrepancies identified and resolved through discussion with an eighth team member (LS). Missing data will be requested from study authors, who will be asked to supply the missing information in de-identified, summarized format. Included studies will be divided between the five reviewers, such that each study has data extracted by three reviewers.

The Cochrane Collaboration's tool for assessing risk of bias will be used to conduct a risk of bias (quality) assessment for individual studies included in the review [25]. As per this tool, criteria for judging risk of bias will be applied (low, unclear, high) to the following domains: selection bias (random sequence generation, allocation concealment); performance bias (blindness of participants and personnel); detection bias (blindness and modality of outcome assessment); attrition bias (incomplete outcome reporting); and reporting bias (selective outcome reporting). A similar approach will be taken to non-randomized studies included in the review, using the eight-item Newcastle-Ottowa Scale as a guide [26]. To determine reporting bias, the ORBIT classification system will be adopted [27] for each included study. In each intervention category (tobacco, physical activity, diet quality, sleep-wake disturbance, alcohol/other drugs), a matrix will be constructed for included studies, which evaluates the reporting of the primary outcome for the review, and other trial outcomes related to that lifestyle behavior. Each reported outcome will be given a rating according to whether the primary outcome/other outcome was reported in full (with statistical analysis results reported in full), not reported at all, or partially reported (e.g., only the $p$ value was provided). For those studies given a "partially reported" or "not reported" rating for the given primary outcomes of interest, the nine-point ORBIT classification system will be applied. Each of these studies will be given a classification of A-I, depending on the clarity with which the primary outcome was measured and analyzed. Clarification will be sought from trial registry information and/or study authors to determine whether a study might be classified as incomplete outcome reporting and at risk of outcome reporting bias (due to a change in outcomes from registered details).

\section{Primary outcomes}

The primary outcomes for the review will be changes in the lifestyle behaviors targeted by the intervention between baseline and the immediate post-intervention follow-up assessment, namely tobacco use, levels of physical activity, diet quality, sleep quality, alcohol use, and illicit drug use. In order to synthesize results across all included studies within a particular lifestyle behavior, a review of reported outcomes for each included study will be conducted by LT and FK-L to determine how the primary outcome of interest is reported for each study in each lifestyle area (e.g., abstinence rates for tobacco use studies versus cigarettes per day, minutes of vigorous exercise per week for physical activity studies versus hours per week of non-leisure sedentary behavior). Collaborating researchers specializing in each of the lifestyle categories will be consulted during this process to ensure that the primary outcome for each category best represents the available data and is relevant and important to the field (tobacco $=\mathrm{AB}$, FK-L, SW; physical activity $=\mathrm{RC}, \mathrm{SD}, \mathrm{SW}$; diet quality $=\mathrm{FJ}$, AT, SW; sleepwake disturbance $=\mathrm{NR}, \mathrm{SH}, \mathrm{TH}, \mathrm{SW}$; alcohol/other drugs $=\mathrm{AB}, \mathrm{FK}-\mathrm{L}, \mathrm{SW})$. SW, a consumer-researcher, will review these primary outcomes for relevance and importance to people experiencing $\mathrm{BD}$.

\section{Data analysis}

The primary analysis (estimated intervention effect) for the review will be based on all studies, regardless of risk of bias, but presented with a description of the risk of bias in individual domains.

We will provide a narrative synthesis of the findings from the included studies, based on the framework reported by Popay et al. [28]. Separate narratives will be presented for each lifestyle domain: tobacco, alcohol, illicit drugs, sleep, physical activity, and healthy eating. We will describe the theoretical basis of the interventions for included studies, which includes discussion about what works, for whom, and why. We will provide summaries of intervention effects in a tabular form, using data from the extraction phase, organized according to primary outcome for that lifestyle domain, and other reported outcomes at all reported timepoints. We will also report on studies in each lifestyle area according to the type of intervention (single, multi-focus, eHealth), target population characteristics (including phase of bipolar disorder), type of outcome (including objective, self-report, clinician-rated), intervention content, and the phase of $\mathrm{BD}$ in which the intervention was delivered (manic/hypomanic, depressed, euthymic). We will calculate and report risk ratios (for dichotomous outcomes such as abstinence from tobacco) 
or standardized mean differences (for continuous outcomes such as minutes of physical activity). Forest plots will be generated for the primary outcome in each lifestyle area.

Where there are two or more randomized controlled trials in a particular lifestyle area, we will carry out a metaanalysis of primary outcome using these studies. We anticipate that there will be limited scope for meta-analysis across all lifestyle domains. The exception may be for smoking cessation where there is likely to be a larger pool of completed studies in BD. The meta-analysis will build on the narrative synthesis in the eligible lifestyle area by combining primary outcomes across studies to provide an overall summary estimate of effect of the lifestyle intervention. Both fixed and random effects models will be applied, stratified by study size, and overall summary estimates of effect added to the forest plots for that lifestyle area.

In interpreting the results of the analysis, the Grading of Recommendations Assessment, Development, and Evaluation system (GRADE) will be applied to determine the quality of evidence for each intervention target reported in the review [29]. The GRADEpro [30] program will be used to facilitate this analysis. We will use the following in grading the quality of the evidence arising from the systematic review for each of the intervention targets in bipolar disorder:

a) High $=$ further research is very unlikely to change our confidence in the estimate of effect.

b) Moderate $=$ further research is likely to have an important impact on our confidence in the estimate of effect and may change the estimate.

c) Low $=$ further research is very likely to have an important impact on our confidence in the estimate of effect and is likely to change the estimate.

d) Very low = any estimate of effect is very uncertain.

\section{Discussion}

In the general population, eliminating health risk behaviors has the potential to prevent $80 \%$ of heart disease, stroke, $80 \%$ of type 2 diabetes, and $40 \%$ of cancers [31]. The same is likely to be true in mental illness. Given the complex nature of bipolar disorder, uncertainty exists about the most effective way to drive improvements in health behaviors, as treatments effective in other populations may not directly and safely translate to this patient population [13]. The planned review will synthesize and evaluate the available evidence regarding the behavioral or psychosocial treatment of lifestyle-related behaviors in people with BD. From this review, we will identify gaps in our existing knowledge and research evidence about the management of unhealthy lifestyle behaviors in people with BD. We will also identify potential opportunities to address lifestyle behaviors in $\mathrm{BD}$, with a view to reducing the burden of physical ill-health in this population.

\section{Additional file}

\section{Additional file 1: Systematic review data extraction form. (PDF 75 kb)}

\section{Abbreviation}

CVD, cardiovascular disease

\section{Acknowledgements}

This systematic review is funded by a UNSW Goldstar grant, awarded after peer review by the National Health and Medical Research Council of Australia project grant scheme. FK-L (APP1008972), MB (APP1059660), and AB receive a fellowship funding from the National Health and Medical Research Council of Australia.

PM is funded through an NHMRC program grant.

\section{Authors' contributions}

This review was conceived by FK-L, who also led the systematic review design, and completed the PROSPERO registration. LKT, JML, TH, LS, FJ, AM, and SW participated in the development and refinement of the methodological approach and in drafting of the study protocol. All authors (FK-L, LKT, JML, TH, $L S, F J, A L B, M B, P B M, R C, N R, S W, S D, C O, A M, C D, A T$, and SH) read, edited, and approved the final manuscript.

\section{Competing interests}

The systematic review team members are co-investigators on a grant application currently under review by the National Health and Medical Research Council (Australia) to deliver a psychological treatment for multiple lifestyle behaviors among smokers with bipolar disorder.

\section{Author details}

${ }^{1}$ National Health and Medical Research Council Centre for Research Excellence in Mental Health and Substance Use, National Drug and Alcohol Research Centre, University of New South Wales, Sydney, Australia. ${ }^{2}$ Priority Research Centre for Translational Neuroscience and Mental Health, The University of Newcastle, Newcastle, Australia. ${ }^{3}$ Black Dog Institute, School of Psychiatry, University of New South Wales, Sydney, Australia. ${ }^{4}$ Department of Psychiatry, Harvard Medical School, Boston, USA. ${ }^{5}$ IMPACT Strategic Research Centre (Innovation in Mental and Physical Health and Clinical Treatment), Deakin University, Waurn Ponds, Australia. ${ }^{6}$ School of Biomedical Sciences and Pharmacy, Faculty of Medicine, The University of Newcastle, Newcastle Australia. ${ }^{7}$ Brain and Mind Institute, University of Sydney, Sydney, Australia.

${ }^{8} \mathrm{CReDITSS}$, Hunter Medical Research Institute, Newcastle, Australia. ${ }^{9}$ The University of Melbourne, Melbourne, Australia. ${ }^{10} \mathrm{School}$ of Psychology, University of Newcastle, Newcastle, Australia.

Received: 14 June 2015 Accepted: 16 June 2016

Published online: 05 July 2016

\section{References}

1. Reisinger-Walker E, McGee RE, Druss BG. Mortality in mental disorders and global disease burden implications: a systematic review and meta-analysis. JAMA Psychiatry. 2015; doi:10.1001/jamapsychiatry.2014.2502.

2. Lawrence $D$, Hancock KJ, Kisley S. The gap in life expectancy from preventable physical illness in psychiatric patients in Western Australia: retrospective analysis of population base registers. Br Med J. 2013;346:f2539.

3. Kessing LV, Vradi E, Andersen PK. Life expectancy in bipolar disorder. Bipolar Disord. 2015;17(5):534-48.

4. Goodrich DE, Kilbourne AM. A long time coming - the creation of an evidence base for physical activity prescription to improve health outcomes in bipolar disorder. Ment Health Phys Act. 2010;3(1):1-3.

5. Kendall T, MOrriss R, Mayo-Wilson E, Marcus E. Assessment and management of bipolar disorder: summary of updated NICE guidance. $\mathrm{Br}$ Med J. 2014;349:95673.

6. Malhi GS, Bargh DM, Mclntyre R, Gitlin M, Frye MA, Bauer MS, et al. Balanced efficacy, safety, and tolerability recommendations for the clinical management of bipolar disorder. Bipolar Disord. 2012;14 Suppl 2:1-21.

7. Tondo L, Isacsson G, Baldessarini RJ. Suicidal behavior in bipolar disorder. CNS Drugs. 2003;17(7):491-511

8. Mitchell PB, Johnston AK, Frankland A, Slade TN, Green MJ, Roberts G, et al, Bipolar disorder in a national survey using the World Mental Health Version 
of the Composite International Diagnostic Interview: the impact of differing diagnostic algorithms. Acta Psychiatr Scand. 2013;127(5):381-93.

9. NMHC. A contributing life, the 2012 National Report Card on Mental Health and Suicide Prevention. Sydney: National Mental Health Commission; 2012

10. Karama S, Ducharme S, Corley J, Chouinard-Decorte F, Starr JM, Wardlaw JM, et al. Cigarette smoking and thinning of the brain's cortex. Mol Psychiatry. 2015;20(6):778-85.

11. Sylvia LG, Friedman ES, Kocsis JH, Bernstein E, Brody BD, Kinrys G, et al. Association of exercise with quality of life and mood symptoms in a comparative effectiveness study of bipolar disorder. J Affect Disord. 2013;151(2):722-77.

12. Jacka FN, Pasco JA, Mykletun A, Williams LJ, Nicholson GC, Kotowicz MA, et al. Diet quality in bipolar disorder in a population-based sample of women. J Affect Disord. 2011;129(1-3):332-7.

13. Kilbourne AM, Goodrich DE, Zongshan L, Post EP, Schumacher K, Nord KM, et al. Randomized controlled trial to assess reduction of cardiovascular disease risk in patients with bipolar disorder: the self-management addressing heart risk trial (SMAHRT). J Clin Psychol. 2013;74(7):e655-62.

14. Stuckler A, McKee M, Ebrahim S, Basu S. Manufacturing epidemics: the role of global producers in increased consumption of unhealthy commodities including processed foods, alcohol, and tobacco. PLoS Med. 2012;9(6):e1001235.

15. Sylvia LG. Association of exercise with quality of life and mood symptoms in a comparative effectiveness study of bipolar disorder. J Affect Disord. 2013;151(2):722-7.

16. Jacka JN, Mykletun A, Berk M. Moving towards a population health approach to the primary prevention of common mental disorders. BMC Med. 2012;10:149.

17. Ng F, Mammen OK, Wilting I, Sachs GS, Ferrier IN, Cassidy F, et al. The International Society for Bipolar Disorders (ISBD) consensus guidelines for the safety monitoring of bipolar disorder treatments. Bipolar Disord. 2009;11:559-95.

18. McGorry P, Alvarez-Jimenez M, Killackey E. Antipsychotic medication during the critical period following remission from first-episode psychosis: less is more. JAMA Psychiatry. 2013;70(9):98-100.

19. Prochaska JJ et al. An online survey of tobacco use, intentions to quit, and cessation strategies among people living with bipolar disorder. Bipolar Disord. 2011;135(5-6):466-73.

20. Wright K, Armstrong T, Taylor AH, Dean S. 'It's a double edged sword': a qualitative analysis of the experiences of exercise amongst people with bipolar disorder. J Affect Disord. 2012;136(3):634-42.

21. Banham L, Gilbody S. Smoking cessation in severe mental illness: what works? Addiction. 2010;105(7):1176-89.

22. Harvey AG, Talbot LS, Gershon A. Sleep disturbance in bipolar disorder across the lifespan. Clin Psychol. 2009;16(2):256-77.

23. Shamseer L, Moher D, Clarke M, Ghersi D, Liberati A, Petticrew M, et al. Preferred reporting items for systematic review and meta-analytic protocols (PRISMA-P) 2015: elaboration and explanation. Br Med J. 2015;349:7647.

24. Liberati A, Altman DG, Tetzlaff JM, Mulrow C, Gotzche PC, loannidis JPA, et al. The PRISMA statement for reporting systematic reviews and metaanalyses of studies that evaluate healthcare interventions: explanation and elaboration. Br Med J. 2009:339:2700.

25. Higgins JPT, Green S. Cochrane handbook for systematic reviews of interventions version 5.1.0 [updated March 2011]. Available from http:// handbook.cochrane.org. Accessed 29 June 2016.

26. Wells GA, Shea B, O'connell D, Peterson JEA, Welch V, Losos M, et al. The Newcastle-Ottawa Scale (NOS) for assessing the quality of nonrandomised studies in meta-analyses 2000. Available from http://www.ohri.ca/programs/ clinical_epidemiology/oxford.asp. Accessed 29 June 2016

27. Dwan K, Gamble C, Kolamunnage-Dona R, Mohammed S, Powell C, Williamson PR. Assessing the potential for outcome reporting bias in a review: a tutorial. Trials. 2010;11:52.

28. Popay J, Roberts H, Sowden A, Petticrew M, Arai L, Rodgers M, et al. Guidance on the conduct of narrative synthesis in systematic reviews: a product from the ESRC Methods Programme. doi:10.13140/2.1.1018.46432006.

29. Guyatt GH, Oxman AD, Vist GE, Kunz R, Falck-Ytter Y, Alonso-Coelle P, et al. GRADE: an emerging consensus on rating quality of evidence and strength of recommendations. Br Med J. 2008:336:924-6.

30. GRADEpro. Computer program on http://www.gradepro.org/]. Version 30 May 2015. In: University M, editor. 2014. Accessed 29 June 2016.

31. Spring B, Moller AC, Coons MJ. Multiple health behaviours: overview and implications. J Public Health. 2012;34(S1):i3-i10.

\section{Submit your next manuscript to BioMed Central and we will help you at every step:}

- We accept pre-submission inquiries

- Our selector tool helps you to find the most relevant journal

- We provide round the clock customer support

- Convenient online submission

- Thorough peer review

- Inclusion in PubMed and all major indexing services

- Maximum visibility for your research

Submit your manuscript at www.biomedcentral.com/submit
Biomed Central 